\title{
Un periodismo en transicion ante el V informe del IPCC, EI acuerdo de París y Los objetivos de desarrollo sostenible
}

institucional.us.es/ambitos/

\section{Rogelio Fernández Reyes}

Grupo de Investigación GREHCCO (Universidad de Sevilla) y Grupo de Investigación MDCS (Universidad Complutense de Madrid)

\section{Juan Carlos Águila Coghlan \\ Grupo de Investigación MDCS (Universidad Complutense de Madrid)}

English Version: Journalism in transition in front of the $\mathrm{V}$ ipcc report, The paris agreement and The sustainable development goals

El Panel Intergubernamental de Cambio Climático (IPCC) reconoce que el cambio climático es claro, inequívoco, ha generado ya impactos en todos los continentes y océanos, y hará falta mitigar y adaptarse para evitar impactos climáticos dañinos (2014). Los Objetivos de Desarrollo Sostenible tienen el cambio climático como uno de los principales retos. El Acuerdo de París (2015) se ha marcado el objetivo de "mantener el aumento de la temperatura media mundial muy por debajo de $2^{\circ} \mathrm{C}$ con respecto a los niveles preindustriales, y proseguir los esfuerzos para limitar ese aumento de la temperatura a $1,5^{\circ} \mathrm{C}$ ". EI IPCC indica que queda poco tiempo para evitar que la media supere los $2^{\circ} \mathrm{C}$ de temperatura con respecto a los niveles preindustriales. En este ensayo se considera que para lograr -o acercarse- al objetivo de que el aumento de temperatura no supere los $1,5^{\circ} \mathrm{C}$ es preciso que prevalezca un cambio transformacional, esto es, sistémico, en un periodo corto de tiempo. Se propone reflexionar cómo procurar un periodismo en transición que, sin renunciar al rigor y a la objetividad, sitúe la sostenibilidad como eje de referencia. Y se reflexiona sobre qué elementos pueden ser interesantes en las prácticas comunicativas ante el V Informe del IPCC, los Objetivos de Desarrollo Sostenible y el Acuerdo de París

Palabras clave: Medios de Comunicación, Periodismo en Transición, Cambio climático, Acuerdo de París, Objetivos de Desarrollo Sostenible.

\begin{abstract}
The Intergovernmental Panel on Climate Change (IPCC) recognizes that climate change is clear, unequivocal, has already generated impacts on all continents and oceans, and mitigation and adaptation will be necessary to avoid harmful climatic impacts (IPCC 2014). The Sustainable Development Goals have climate change as one of the main challenges. The Paris Agreement (2015) has set itself the goal of "keeping the average global temperature rise well below $2^{\circ} \mathrm{C}$ above pre-industrial levels and continuing efforts to limit that temperature rise to $1,5^{\circ} \mathrm{C}$. The IPCC indicates that there is little time left to prevent the average temperature rises above $2^{\circ} \mathrm{C}$ from pre-industrial levels. In this essay, it is considered that in order to achieve -or approachthe objective of increasing the temperature not to exceed $1.5^{\circ} \mathrm{C}$, a transformational change, that is, systemic, must take place over a short period of time. It is proposed to reflect on how to seek journalism in transition that, without renouncing rigor and objectivity, places sustainability as a reference axis. And we reflect on what elements may be interesting in communicative practices before the V IPCC Report, the Sustainable Development Goals and the Paris Agreement.
\end{abstract}

Keywords: Media, Transition Journalism, Climate Change, Paris Agreement, Sustainable Development Goals. 


\section{Introducción}

Nos encontramos en un momento histórico excepcional ante la realidad del cambio global [01], un reto de una gran dimensión que requiere una gran respuesta. El cambio global muestra que el ser humano está modificando el estado de estabilidad relativa de la biosfera de los últimos miles de años y forzando su variabilidad natural. El crecimiento de la extralimitación de la especie humana en varios umbrales ambientales (Rockström et al, 2009) conlleva el aumento del desequilibrio en la biosfera en la presente etapa del Antropoceno (Crutzen y Stoermer, 2000). Hoy el cambio climático [02] es una manifestación de este cambio global, llamado a ser su principal motor, determinando cada vez en mayor medida la alteración del funcionamiento y de los servicios de los ecosistemas terrestres a escala mundial (Duarte, 2006: 93).

El Memorando de Estocolmo (2011), firmado por una veintena de premios Nobel, reconoció al ser humano como principal causante del cambio climático, cuyas acciones colectivas pueden tener consecuencias irreversibles para el planeta. "No podemos seguir este camino y necesitamos ponernos en acción rápido", concluía el Nobel de Química, Mario Molina. La transición hacia otras formas de vida sostenibles "plantea un ejercicio de revisión profunda sobre el momento histórico en el que nos encontramos requiriendo una cosmovisión distinta a la que nos ha traído a la crisis", expone María Novo (2016: 233).

Como contrapunto a la extralimitación que parte de la humanidad está ejerciendo se encuentra la apuesta por una transición [03] que otorga el protagonismo a la sostenibilidad, en un marco de sistemas adaptativos complejos. Se trata de retroceder en los límites transgredidos, evitar cruzar más límites, o minimizar los efectos de los umbrales traspasados, que permita a las nuevas generaciones y el resto de las especies poder disfrutar del sistema Tierra lo menos trastocado posible con respecto a los últimos miles de años. Consideramos que se precisa de una desaceleración, un decrecimiento material y energético en parte de la población del planeta para crecer en humanidad, aceptando los límites del crecimiento, realidad compleja en cuanto a la distribución de los recursos disponibles y a la responsabilidad de la situación actual.

La práctica periodística refleja y participa (Rojo y Maestre, 2005) en la representación social de la sostenibilidad y en la aportación de soluciones. Los medios de comunicación, por tanto, frenan o facilitan el abordaje de un cambio cultural como el que entendemos que se requiere. El presente ensayo es una actualización resumida, acompañada de nuevos contenidos, del artículo "En búsqueda de un periodismo en transición" (FernándezReyes, 2016b). En él, se trataba de justificar la necesidad de un cambio sistémico en el ejercicio periodístico y se abordaban algunas funciones y disfunciones del periodismo en torno a la sostenibilidad.

Va destinado a profesionales y estudiantes de la profesión periodística, así como a investigadores de la comunicación interesados en la reacción frente a la extralimitación del ser humano en la biosfera. No pretende convencer sino reflexionar en voz alta en la búsqueda de un periodismo en transición y quizás facilitar que otros encuentren sus propias conclusiones ante el reto crucial al que nos enfrentamos. Para muchos contextos podrá resultar imposible o utópico. Pero, ¿no es más utópico pensar que podemos seguir con las dinámicas de crecimiento sin acabar en un colapso, en una implosión de la civilización actual?

Nos centraremos, principalmente, en el análisis de elementos de transición en el campo de los contenidos periodísticos. Las limitaciones nos impiden abordar elementos tan importantes como las estructuras periodísticas o la realidad de la profesión y de los profesionales, que serán imprescindibles estudiar. Nos detendremos concretamente en el objetivo de reaccionar ante el incremento de temperatura acordado en el Acuerdo de París (COP 21), partiendo de la base científica del V Informe del IPCC, en línea con los Objetivos de Desarrollo Sostenible.

\section{EI V Informe del IPCC, el Acuerdo de París y los Objetivos de Desarrollo Sostenible en el paradigma del crecimiento}

EI V Informe del Panel Intergubernamental de Cambio Climático (IPCC, 2013-2014) de la ONU, confirma una 
serie de conclusiones referidas a la realidad del cambio climático, sus causas, sus consecuencias y sus soluciones: a) "El calentamiento del sistema climático es inequívoco" (IPCC, 2013: 4); b) "La influencia humana en el sistema climático es clara" (IPCC, 2013: 15); c) "En las últimas décadas, los cambios en el clima han causado impactos en los sistemas naturales y humanos en todos los continentes y océanos" (IPCC, 2014a: 4); d) "Las sociedad mundial tendrá que mitigar y adaptarse al cambio climático si quiere evitar con eficacia los impactos climáticos dañinos" (IPCC, 2014b: 50).

El Acuerdo de París (2015), declaró perseguir el objetivo climático de: "Mantener el aumento de la temperatura media mundial muy por debajo de $2^{\circ} \mathrm{C}$ con respecto a los niveles preindustriales, y proseguir los esfuerzos para limitar ese aumento de la temperatura a $1,5^{\circ} \mathrm{C}$ ". ¿Qué supondría que todos los Estados cumplieran sus promesas y atendieran a los compromisos aportados en París? Christiana Figueres, Secretaria Ejecutiva de la CMNUCC ${ }^{[04]}$, remitió a $2,7^{\circ} \mathrm{C}$ como cifra que acompañaría al cumplimiento de las promesas nacionales (UNFCCC, 2015). Pero si se tienen en cuenta otros estudios de contribuciones determinadas a nivel nacional (INDCs), el resultado es de una horquilla entre 2,7 y $3,7^{\circ} \mathrm{C}$ [05].

En septiembre de 2015, representantes de 193 países adoptaron un compromiso histórico al aprobar los 17 Objetivos de Desarrollo Sostenible. La ONU, en su página Web, concentraba estos objetivos en "erradicar la pobreza, proteger el planeta y asegurar la sostenibilidad" [06]. Los Objetivos de Desarrollo del Milenio la ONU (2000-2015) pasó de tener 1 objetivo (de 8) relacionado con la sostenibilidad a tener en los Objetivos de Desarrollo Sostenible (2015-2030) a 11 objetivos (de 17) en los que se hace mención al término sostenible, a lo que hay que sumar su presencia en el título y un objetivo específico sobre el reto que nos ocupa: "Adoptar medidas urgentes para combatir el cambio climático y sus efectos".

¿En qué paradigma se enmarcan el V Informe del IPCC, el Acuerdo de París y los Objetivos de Desarrollo Sostenible? En el paradigma del crecimiento. EI V Informe del IPCC identifica dos principales motores del cambio climático: el crecimiento económico y el crecimiento demográfico (IPCC, 2014c: 5). ¿Es posible abordar el cambio climático sin ir a las raíces, sin cambiar el modelo económico prevaleciente y las tendencias demográficas?

EI IPCC trabaja sobre los requerimientos de los países y éstos no se plantean premisas que comprometan el desarrollo (por ejemplo: IPCC, 2014b: 20). Los científicos no incluyen escenarios de decrecimiento material en los países de mayor impacto en la Tierra. ¿Es posible un cambio urgente en la gobernanza climática sin comprometer el desarrollo? ${ }^{[07]}$ Entendemos que el desarrollo ha de satisfacer las necesidades sociales dentro de los límites que impone la biosfera. El considerar que la biosfera ha de satisfacer las necesidades del ser humano dentro de los límites que impone el desarrollo forma parte de una limitada actitud antropocentrista excluyente y corto-placista, que acaba volviéndose en contra de su legítima aspiración. [08]

Los contenidos más sugestivos del Acuerdo de París se encuentran en el preámbulo, que carece de fuerza legal. Aquí se reconoce que el cambio climático es "una amenaza apremiante y con efectos potencialmente irreversibles para las sociedades humanas y el planeta", que "requerirá fuertes reducciones de las emisiones mundiales" y la "urgencia" de hacerles frente. Sin embargo, el Acuerdo en sí no presenta un cambio sustancial. Estuvo limitado por fuertes intereses políticos y económicos, los cuales retiraron el término descarbonización, en un marco de crecimiento económico incuestionado. Por ello se pasó de la figura de Protocolo a la de Acuerdo, que rebajó la fuerza legal internacional del texto (Fernández-Reyes, 2016a).

El crecimiento sigue siendo una premisa indiscutible en los Objetivos de Desarrollo Sostenible. No se cuestiona el modelo hegemónico neoliberal de producción y consumo (Meira, 2015: 58). Como expone el profesor Meira, desde el prisma socio-ambiental, el problema no se encuentra en lo acertado de los objetivos definidos o en la posibilidad de su cumplimiento en los plazos estimados, como en el modelo de desarrollo que enmarca. Se considera que, "sin crecimiento, no será posible erradicar la pobreza y generar bienestar, ni liberar los recursos suficientes -tecnológicos, culturales, económicos- para alcanzar una gestión sostenible de la biosfera. El crecimiento aparece, por lo tanto, como una conditio sine qua non del desarrollo y de la sostenibilidad" (p. 59).

Edgar Morin defiende que la idea de desarrollo es una idea subdesarrollada (Morin, 2011: 27). Como expone el filósofo y sociólogo francés, la idea fija del crecimiento, “¿no debería sustituirse por un concepto complejo que 
comportase crecimientos, decrecimientos y estabilizaciones diversas"? (p. 25)

¿Y qué probabilidades hay de quedarnos en un incremento de la media global de " $1,5^{\circ} \mathrm{C}$ " o "muy por debajo de $2^{\circ} \mathrm{C}$ "? Desde el ámbito científico se considera que, si no hay un drástico recorte cuantificado de emisiones para el 2050, los objetivos de aumentos de temperatura "por debajo de $2^{\circ} \mathrm{C}$ " ó " $1,5^{\circ} \mathrm{C}$ " es un ejercicio de voluntarismo. ${ }^{[09]}$ ¡Habría que llegar al pico de emisión en 2020 para lograr que el aumento no supere los "1,5 C"! Ante estos objetivos climáticos, ¿qué tipo de cambio es preciso?

\section{Periodismo en transición ante el V Informe del IPCC, el Acuerdo de París y los Objetivos de Desarrollo Sostenible}

El informe de Síntesis El medio ambiente en Europa. Estado y perspectivas 2015, de la Agencia Europea de Medio Ambiente (AEMA) recogía:

"Vivir bien sin rebasar los límites ecológicos requiere transiciones fundamentales en los sistemas de producción y consumo, los responsables últimos de las presiones medioambientales y climáticas. La propia naturaleza de estas transiciones hará necesarios cambios de gran calado en las instituciones, las prácticas, las tecnologías, las políticas, los estilos de vida y el pensamiento predominante" (p. 14).

A día de hoy se puede afirmar que la virtualidad mediática está alejada de la realidad biofísica. Los medios de comunicación pueden recortar esta lejanía o fomentarla. La búsqueda de un periodismo en transición se plantea como una opción voluntaria -que puede tornarse en forzada involuntariamente si traspasamos umbrales climáticos que ocasionen cambios a gran escala-, una apuesta cívica ante el desequilibrio que está generando el ser humano en la biosfera. Es una interpretación y a la vez una propuesta que parte de la consideración de que la situación de extralimitación del cambio climático requiere un cambio cultural, que a su vez reclama un cambio mediático.

Periodismo en transición se podría definir como la percepción teórica y el ejercicio periodístico que, ante el cambio global, y principalmente el cambio climático, opta por un cambio cultural en el que se prioriza la sostenibilidad como eje. Se asume como una función social de los medios de comunicación y un requerimiento legítimo de la sociedad. Se justifica por el cuidado de la especie humana y de las generaciones futuras, y el cuidado del resto de las especies y los ecosistemas, considerando que las demás especies tienen un valor per se.

Ante el reto del cambio climático, un periodismo en transición arranca de un punto de partida que es la extralimitada concentración de gases de efecto invernadero ${ }^{[10]}$ y su relación con el aumento de la temperatura, hoy mostrada con robustez desde el ámbito científico. El punto de llegada es el freno y retroceso en la concentración de CO2-eq en la atmósfera y en el aumento de la temperatura. Concretamente, el objetivo de un incremento de temperatura muy por debajo de $2^{\circ} \mathrm{C}$, persiguiendo que no supere $1,5^{\circ} \mathrm{C}$.

A continuación, se sugieren algunos puntos que quizás puedan resultar de interés en la búsqueda de un periodismo en transición sobre el abordaje de la comunicación del cambio climático y de las alternativas tras los resultados del V Informe del IPCC, el Acuerdo de París y los Objetivos de Desarrollo Sostenible.

\section{a) Un tratamiento cuantitativo y cualitativo mayor y mejor}

Si el cambio climático se ha posicionado como tema prioritario en la agenda internacional en París, con la mayor afluencia histórica de jefes de Estado, es oportuno un mayor tratamiento mediático [11]: facilitando el debate, visibilizando la movilización ciudadana, las experiencias alternativas ${ }^{[12]}$, los gestos de voluntad y práctica política, el reconocimiento de la dimensión real del problema, el pronunciamiento de líderes de distintos ámbitos, las iniciativas de compromiso común, los sucesivos informes científicos. Está injustificado que la proporción de presencia mediática del cambio climático no sea paralela a la robustez científica y a la percepción del riesgo: 
Cobertura en prensa española de los términos cambio climático o calentamiento global (enero 2000mayo 2017): datos por cabecera y total

\section{0-2017 Spain Newspaper Coverage of Climate Change or Global Warming}

800

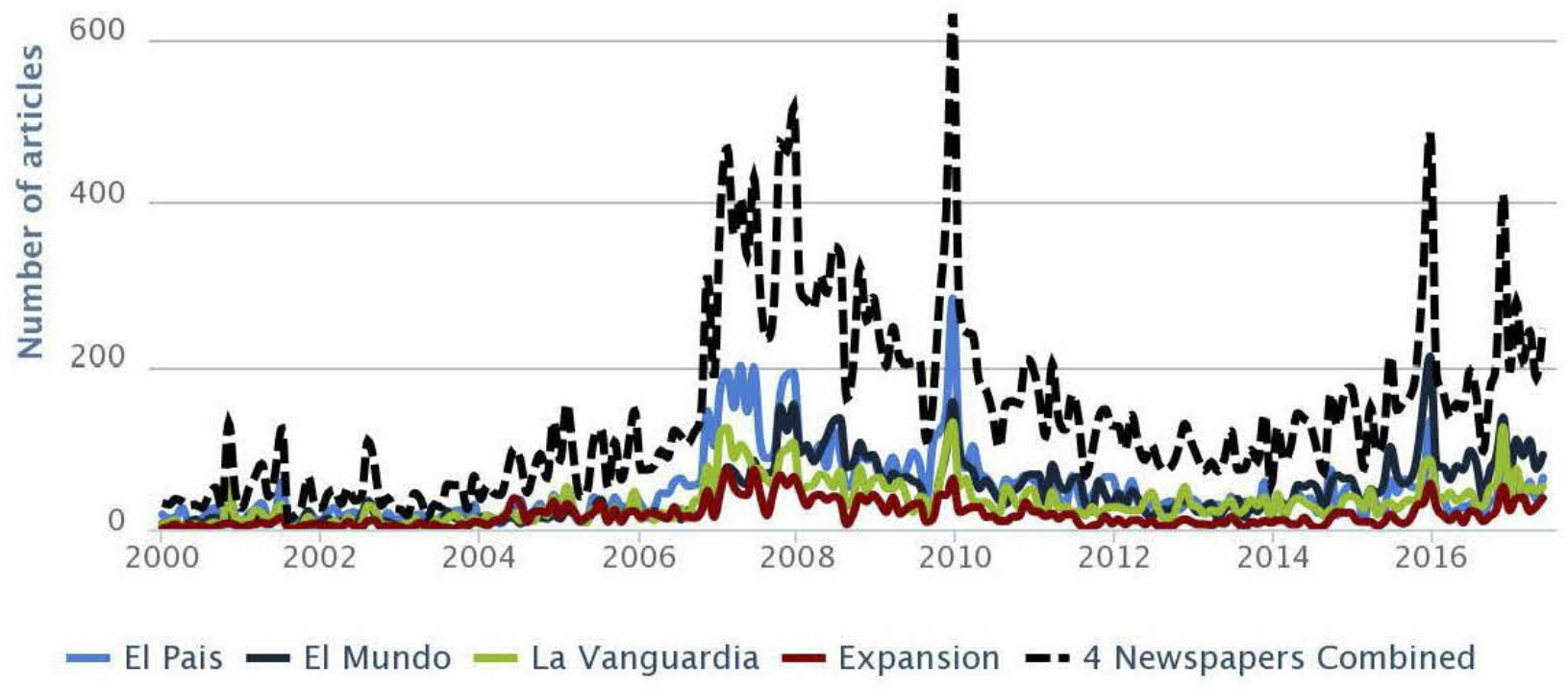

Gráfico 1. Fernández-Reyes, Rogelio, 2017. Spain Newspaper Coverage of Climate Change or Global Warming, 2000-2017. Universidad de Sevilla, Web. [Date of access.]

http://sciencepolicy.colorado.edu/media_coverage/spain.

En cuanto al tratamiento cualitativo, puede ser oportuno tener en cuenta que los relatos periodísticos sobre cambio climático son diferentes si se elaboran desde un paradigma productivista que si se elaboran desde un paradigma ecológico. Ello pone en cuestionamiento la supuesta asepsia. Estas diferencias se pueden percibir, entre otros elementos, en: una mayor o menor cobertura de un tema, selección de actores (instituciones, expertos y contra-expertos), los argumentos (en los que se interpretan y construyen la realidad), la propuesta de tomas de medidas (campos de acción o de inacción), la atribución de responsabilidades, el reconocimiento de las consecuencias, el grado de reconocimiento o cuestionamiento de las afirmaciones científicas o de las distintas fuentes (Fernández-Reyes, 2010: 16b). Sería oportuno avanzar en la integración del paradigma ecológico a la hora de elaborar los relatos.

\section{b) Vincular el objetivo climático con la reducción y la descarbonización.}

El debate sobre el objetivo climático no debe sustituir lo verdaderamente importante, que es la reducción de emisiones (Boykoff et al, 2010). La rebaja del objetivo climático en el Acuerdo de París de $2^{\circ}$ a $1,5^{\circ} \mathrm{C}$ ha sido un logro de la movilización ciudadana y política, ligado directamente al término "descarbonización". EI V Informe del IPCC habla claramente de que para mitigar el cambio climático será necesario descarbonizar el sector energético ${ }^{[13]}$. Además de ser un término usado en el V Informe del IPCC, la ONU lo incluyó en los borradores de la Cumbre de París, pero se retiró a instancias de países productores de petróleo. Al final, el Acuerdo de París derivó en el término "equilibrio", amparado en los mecanismos de secuestro y almacenamiento de carbono [14].

Ante ello consideramos que es irrenunciable el uso y la práctica del término "descarbonización" para lograr el objetivo de temperatura acordado en París. Una cultura baja en carbono está confrontada irremediablemente 
con los intereses empresariales, políticos y sociales en torno a los combustibles fósiles. También con los hábitos de vida consumistas adictos al carbono, que habrán de atravesar una desintoxicación. Y es imprescindible introducir el debate sobre las medidas para el transporte marítimo y aéreo, eliminadas de los borradores del Acuerdo. Se trata de dos de los sectores que más emisiones generan: el $10 \%$ de las emisiones mundiales.

\section{c) Vincular el cambio climático con los flujos migratorios}

Una de las consecuencias del cambio climático son las migraciones. Se trata de un tema aún poco investigado y poco visibilizado que tiene un gran interés (Rivillo, 2015). Si bien no se puede atribuir que el cambio climático sea el causante de éstas, sí se puede defender que, cada vez más, el cambio climático es un factor fundamental en las mismas. Eventos meteorológicos extremos desplazaron de sus hogares a 184 millones de personas entre 2008 y 2014 . [15]

El marco ético y el valor del bien común favorecen el tratamiento de este tema. No hay que olvidar que naciones y ciudadanos con baja producción de emisiones son los que más sufren y sufrirán, junto a las nuevas generaciones, otras especies y ecosistemas, el impacto producido por naciones y ciudadanos responsables de emisiones altas. Países con pocas emisiones de gases de efecto invernadero son, paradójicamente, especialmente vulnerables a los efectos del calentamiento global. Africa sólo es responsable del $3 \%$ de las emisiones de $\mathrm{CO} 2{ }^{[16]} \mathrm{y}$, sin embargo, 36 de los 50 países que más sufren el cambio climático se encuentran en este continente [17]. Esto es, el $72 \%$ de los 50 países más afectados son africanos. Africa soporta la principal carga del calentamiento global (Ruiz-Cabrera, 2017).

\section{d) Favorecer el debate sobre el cambio en tres motores:}

EI IPCC considera el crecimiento económico y el crecimiento demográfico como los principales motores del cambio climático. Si los desgranamos un poco más podemos considerar que entre dichos motores se encuentran el crecimiento de la población, el modelo de producción y consumo, y el modelo energético. Es importante el debate en la transformación de cada uno.

- Crecimiento de la población: El informe de la ONU "Proyecciones poblacionales de las Naciones Unidas" indica que la población superará los 11 mil millones en 2100. La población mundial pasará de los actuales 7,3 mil millones de personas a 9,7 mil millones en 2050 y 11,2 mil millones a finales de siglo [18]. Motivos ideológicos y religiosos han tenido un peso fuerte en la claudicación o la poca atención a este motor, lo cual es preciso revertir.

- Modelo de producción y consumo: los medios pueden incentivar un patrón cultural de producción y consumo sin límites o bien otro que lo cuestione y promueva la sostenibilidad. Según el Worldwatch Institute, la inmensa mayoría de los medios de comunicación refuerzan actualmente un patrón cultural consumista -a través de la publicidad [19], la venta de productos y gran parte de sus contenidos [20]-, aunque se están realizando esfuerzos en todo el mundo para aprovechar su extraordinario alcance y potencial para promover culturas sostenibles (2010: 285). Otro debate interesante puede ser el que se plantea si los mercados de carbono son compatibles o no con la reducción requerida.

- Modelo energético: El sector de suministro energético el mayor contribuyente de emisiones de gas invernadero a nivel global en 2010 (IPCC, 2014b: 7). Es sobre este motor sobre el que se ha centrado el mayor interés en el Acuerdo de París. Si bien, no es la única pata en un cambio cultural [21].

\section{e) Verificar la sintonía o no entre los objetivos planteados y las contribuciones comprometidas del Acuerdo de París}

Se reconoce una inconsistencia entre los objetivos y los compromisos planteados en la capital francesa (Fernández-Reyes, 2016a: 112). A la par, el ritmo del impacto humano no cesa de aumentar en el sistema climático ${ }^{22}$. Es preciso analizar el nivel de responsabilidad/vaguedad de los compromisos nacionales, políticos, económicos y sociales, así como el acierto o no del calendario marcado y las revisiones. Dado el cariz que está tomando el riesgo asociado del cambio climático, está justificada la recuperación de la función 
periodística de "perros guardianes" del bien común. Existen perros guardianes de los grandes intereses dentro de las grandes estructuras mediáticas. Estructuras que, como expone el profesor Reig, mientras más fuertes son, más limitan a los medios de comunicación por presiones que reciben y por las autocensuras que se imponen a tenor de los intereses que guardan en su parte trasera, invisible para casi todos. [23] En la sociedad del gran espectáculo la invitación es a conectarse y consumir (Berenguer, 2002: 7-8). Mientras, países pobres pagan ya un precio brutal por el cambio climático ${ }^{[24]}$.

El movimiento climático ha adquirido una dimensión notable. Ha sido impulsor decisivo de que el objetivo climático se rebaje a $1,5^{\circ} \mathrm{C} .{ }^{[25]}$ Es un agente social excelente para denunciar la inconsistencia de los compromisos adquiridos a día de hoy y se mantendrá señalando lo heroico o lo villano ${ }^{[26]}$ en las iniciativas de acción o inacción climática. Dicho movimiento climático está liberado de los intereses políticos que tiene cada país, así como de los intereses empresariales, por lo que sus reclamaciones por el bien común tienen un peso ético más depurado que el de otras voces. Junto al ámbito científico es un marco y una fuente atractiva para los medios de comunicación.

\section{f) Priorizar la atención sobre la mitigación y la adaptación transformacional}

La adaptación y la mitigación son dos estrategias complementarias de respuesta al cambio climático. Ambas respuestas pueden reducir y manejar los riesgos del cambio climático. El IPCC considera las respuestas de la mitigación y la adaptación, claudicando hasta el momento del protagonismo de la evitación y la restauración. [27] Para lograr los objetivos planteados en el Acuerdo de París observamos el peso específico que adquiere la mitigación debido a que presenta un potencial particularmente mayor. El Artículo 7.4 del Acuerdo expone: "Las Partes reconocen que la necesidad actual de adaptación es considerable, que un incremento de los niveles de mitigación puede reducir la necesidad de esfuerzos adicionales de adaptación". Cuanto antes se hagan los esfuerzos de mitigación más posibilidades habrá de lograr mantener el incremento de temperatura "muy por debajo de los $2^{\circ} \mathrm{C}$ " o en " $1,5^{\circ} \mathrm{C}$ " y menos adaptación será necesaria. "De demorarse las medidas de mitigación podrían verse limitadas las posibilidades de trayectorias resilientes al clima en el futuro" (IPCC, 2014b: 32).

La mitigación y la adaptación son complementarias en una complejidad de interacciones. Sin embargo, hay un riesgo de exceso de predominio de la adaptación, en cuanto permite seguir con el modelo de producción y consumo, mientras que la mitigación lo traba. Puede ser oportuno estar atento a un posible desplazamiento del negacionismo y del escepticismo hacia el adaptacionismo, deformación de la adaptación como respuesta al mantenimiento del status quo.

La adaptación puede ser progresiva o transformacional, dependiendo si la virulencia de los cambios es suave o fuerte. Consideramos, de manera especial, poner la atención en la adaptación transformacional por las tendencias de emisiones a día de hoy y por definirse en el ámbito sistémico.

\section{g) Informar sobre el efecto multiplicador y de la no linealidad del cambio climático}

A día de hoy desconocemos la existencia de algún estudio que profundice en el factor de multiplicación del aumento de temperatura en los grados centígrados en España, el cual depende de numerosas circunstancias. Pero sí se puede afirmar que el incremento de temperatura por el cambio climático en España aproximadamente dobla al global, si bien ese número varía entre las diferentes estaciones (sobre todo invierno y verano), altitudes-latitudes y diferentes alcances temporales.

Los modelos se plantean una linealidad de calentamiento global que no tienen en cuenta los puntos de inflexión [28]. Es bueno no olvidar las referencias a cambios bruscos y abruptos que han ido apareciendo en todos los informes del IPCC:

I Informe del IPCC: "Podrán ser bruscos, no graduales”, p. 62 Resumen General (1990) II Informe del IPCC: "Puede haber sorpresas no lineales", p. 6 Segunda Evaluación (1995) III Informe del IPCC: "Cambios repentinos, no lineales y de graves resultados", p.16 Síntesis (2001) IV Informe del IPCC: "Cambios abruptos e irreversibles a largo plazo", p. 78 Síntesis (2007) 


\section{h) Favorecer el debate del cambio de modelo civilizatorio ante el modelo de crecimiento hegemónico insostenible}

Numerosos estudios sobre la cobertura del cambio climático en España muestran la prevalencia de un desequilibrio entre la información de los impactos, la de atribución de responsabilidades y las soluciones, siendo mayor la atención a los impactos que a los otros dos vectores. Si nos centramos en las soluciones creemos oportuno el debate sobre la necesidad de un cambio de modelo cultural: pasar de la sociedad de consumo creciente e ilimitado a una sociedad con la sostenibilidad como eje. Si es así, este debate apenas está presente en la estructura de los medios de comunicación y en su discurso hegemónico. Y el reto es impresionante (Fernández-Reyes, Teso y Piñuel, 2013). Tampoco está presente en el marco científico, el cual está apuntando cada vez más a poner en cuestión la vigencia del sistema social imperante, basado en el objetivo de un crecimiento insostenible. "Pero este es un discurso que todavía no es hegemónico y todavía ni siquiera es un discurso canónico en la comunidad científica" (Piñuel, 2016: 27).

El terreno está abonado para un gran cambio cultural, un nuevo paradigma, con nuevos estilos de vida. Es preciso dar un salto hacia el buen vivir, reconociendo los límites del planeta y la necesidad de reducir el impacto. "Son necesarias estrategias narrativas que promuevan el cambio social hacia un nuevo paradigma" (Teso, 2015).

\section{i) Difundir las herramientas: huella de carbono y la huella ecológica}

Las herramientas de la huella ecológica ${ }^{[29]}$ y la huella de carbono ${ }^{[30]}$ son dos instrumentos válidos para el análisis de la realidad del impacto del ser humano ligado a su responsabilidad. Dentro de la huella ecológica ha ido adquiriendo un protagonismo especial la huella de carbono: si en 1961 suponía el $43 \%$ de la huella ecológica, en 2012 supuso el 60 \% [31]. En la mayoría de los años ha habido una tendencia al alza del peso del carbono en la huella ecológica. La causa principal ha sido la quema de combustibles fósiles: carbón, petróleo y gas natural.

En la actualidad, la especie humana necesita más de un planeta y medio para mantener la demanda existente. En 2013, la humanidad necesitó la biocapacidad equivalente de 1,7 planetas Tierra para atender su demanda [32]. No obstante, es oportuno diferenciar entre los países más emisores y las emisiones per cápita. Lo que muestra una responsabilidad bien diferenciada.

\section{Conclusión y discusión}

El cambio climático está adquiriendo un cariz especial de la emergencia mundial, por lo que la práctica comunicativa asume una gran función social en el abordaje de este reto. La ciencia mostró conclusiones nítidas sobre el cambio climático. EI V Informe del IPCC (2014) confirmó que el calentamiento del sistema climático es claro, inequívoco, que ha generado ya impactos en todos los continentes y océanos, y que hará falta mitigar y adaptarse para evitar impactos climáticos dañinos. La Cumbre de París, en diciembre de 2015, contó, de manera inaudita, con la presencia de 150 jefes de Estado, lo que muestra el nivel de reconocimiento del desafío. Por otro lado, la sostenibilidad y el cambio climático se incluyeron de manera notoria en las prioridades de la ONU en los Objetivos de Desarrollo Sostenible para los años 2015-2030.

A pesar del reconocimiento científico y político de la gravedad del cambio climático no se logra revertir el problema. Es más, sigue acentuándose [33]. Frenar el cambio climático se convierte en uno de los principales retos de la especie humana en este contexto histórico. La extralimitación y la posibilidad de colapso ambiental en el horizonte, visto desde el análisis sistémico, requieren un reajuste que se puede traducir en un cambio cultural. Nos embarcamos en la mayor amenaza de la especie humana. Estamos viviendo un momento histórico decisivo, un desafío colosal no solo para la civilización actual sino para la especie humana y el resto de especies "compañeras de viaje". Hay que modificar la curva de emisiones en un plazo de tiempo muy corto. Ello justifica la indagación de un periodismo en transición como función social de los medios de comunicación y 
como requerimiento legítimo de la sociedad.

La búsqueda de un periodismo en transición aspira a tomar forma en multitud de escenarios distintos, motivando una continua y creativa reelaboración de conceptos y de prácticas, con un abordaje multisectorial y multiescala sobre el cambio climático. Aun así, no es nada nuevo. De hecho, se viene fraguando desde hace tiempo en ciertas prácticas comunicativas. Si hay algo nuevo bajo el sol es el grado de emergencia planetaria que nos señalan los procesos de extralimitación del cambio climático, los cuales motivan a que esté más justificado avanzar por este tipo de ejercicio periodístico. Es por lo que consideramos que la búsqueda de un periodismo en transición es una de las claves de la investigación y del ejercicio periodístico de nuestro tiempo para atender el objetivo climático de París, los Objetivos de Desarrollo Sostenible y los riesgos del cambio climático de los que está alertando la ciencia.

\section{Referencias bibliográficas}

BERENGUER Jané, Mariano (2002). Introducción al Periodismo Científico. Sevilla: Padilla Libro Editores \& Libreros

BOYKOFF M.T., MARCO D., RANDALLS S. (2010). "Discursive stability meets climate instability: a critical exploration of the concept of "climate stabilization" in contemporary climate policy". Global Environ Change 2010, 20: 53-64.

CAMBIO CLIMATICO: Mitigación. Guía resumida del Quinto Informe de Evaluación del IPCC, Grupo de Trabajo III (2014). Ministerio de Agricultura Alimentación y Medio Ambiente

CAMBIO GLOBAL EN ESPAÑA 2020/2050. Energía, Economía y Sociedad (2011). Asturias: Ed. CCEIM y Fundación Conama

CAPRA, F. (1982). El punto crucial. Ciencia, sociedad y cultura naciente. Barcelona: Integral

CRUTZEN, P. J. y STOERMER, E. F. (2000). "The Antropocene" en Global Change Newsletter, 41

DUARTE, Carlos M. (coord.), (2006). Cambio global. CICS, Madrid

FERNANDEZ-REYES, R., TESO, G. y PIÑUEL, JL. (2013). "Propuestas de soluciones en la comunicación del cambio climático", en Razón y Palabra 84

FERNANDEZ-REYES, Rogelio (2010). "Reconocimiento y cuestionamiento mediático del cambio climático en España”, en Contribuciones a las Ciencias Sociales, diciembre 2010

- (2016a). "El Acuerdo de París y el cambio transformacional", en Papeles de relaciones ecosociales y cambio global $\mathrm{n}^{\circ} 132$, pp. 101-114

- (2016b). En búsqueda de un periodismo en transición ante el cambio climático, Razón y Palabra 93, pp 776806

- (2017) Spain Newspaper Coverage of Climate Change or Global Warming, 2000-2017. Universidad de Sevilla, Web. [Date of access.] http://sciencepolicy.colorado.edu/media_coverage/spain, consultado el 1 de junio de 2017

HERRERO, Y., CEMBRANOS, F. y PASCUAL, M. (coords.) (2011). Cambiar las gafas para mirar el mundo. Una nueva cultura de la sostenibilidad. Madrid: Libros en Acción

HOPKINS, Rob, (2008). The Tansition Handbook. From oil dependency to local resilience. Totnes: Green Books. IPCC (2013). AR5 Working Group I. Climate Change 2013: The Physical Science Basis. 
- (2014a). AR5 Working Group II. Climate Change 2014: Impacts, Adaptation, and Vulnerability

- (2014b). AR5 Working Group III. Climate Change 2014: Mitigation of Climate Change

- (2014c). AR5. Informe de Síntesis. Cambio climático 2014

MEADOWS, Donella y RANDERS, Jorgen y MEADOWS, Dennis (2006). Los límites del crecimiento: 30 años después. Barcelona: Galaxia Gutenberg

MEIRA, Pablo Ángel (2015). "De los Objetivos de Desarrollo del Milenio a los Objetivos para el Desarrollo Sostenible: el rol socialmente controvertido de la educación ambiental”. Educació Social. Revista d'Intervenció Socioeducativa, 61 , p. 58-73

MORIN, Edgar (2011). La Vía. Para el futuro de la humanidad. Barcelona: Paidós

NOVO, María (2016). "La necesaria transición personal: no se cambia la vida sin cambiar la propia vida", en PRATS, Fernando, Herrero, Yayo y Torrero, Alicia La gran encrucijada. Sobre la crisis ecosocial y el cambio de ciclo histórico. Madrid: Libros en Acción

PIÑUEL, José Luis (2016), "Discursos mediáticos y representaciones sociales sobre el cambio climático" Redes.com $\mathrm{n}^{\circ} 13$

RASKIN, Paul et al (2006). La Gran Transición: la promesa y la atracción del futuro. CEPAL: Santiago de Chile REES, W. y WAKKERNAGEL, M. (1996). Our Ecological Footprint. Reducing Human Impact on Earth. New Society Publishers, Canada. Traducido al español en Rees, W. y Wackernagel, M. (2001). Nuestra huella ecológica. Reduciendo el impacto humano sobre la Tierra, Santiago de Chile: LOM ediciones

REIG, Ramón (1994). La mente global. Un estudio sobre estructura y análisis de la información. Madrid: Libertarias/Prodhufi

- (1998). Medios de comunicación y poder en España. Prensa, radio, televisión y mundo editorial. Barcelona: Paidós Papeles de Comunicación 21

RIVILLO (2015). Refugiados climáticos y el cambio social en territorios frontera. Tesis doctoral defendida en la Facultad de Ciencias de la Información de la Universidad Complutense de Madrid

ROCKSTRÖM et al., (2009). "A safe operating space for humanity", Nature 461: 472-475

ROJO, T. y MAESTRE, J. (2005). "El papel de los Medios de Comunicación en el cambio social ¿cronista o protagonista?”, en GOMEZ y MENDEZ, José Manuel (ed. lit.), Entre la formación y la comunicación, pp. 250-277

RUIZ-CABRERA, Sebastián (2017). "A fuego lento. Efectos del cambio climático en Africa", revista Pueblos n 73. Madrid: Paz con dignidad

TESO, G (2015). Comunicación y representaciones del cambio climático: el discurso televisivo y el imaginario de los jóvenes españoles. Tesis doctoral, Facultad de Ciencias de la Información de la Universidad Complutense de Madrid.

WORLDWATCH INSTITUTE (2010). La situación del mundo 2010: Cambio cultural. Del consumismo hacia la sostenibilidad. Barcelona: Icaria

[01] Por cambio global se entiende "el conjunto de cambios ambientales afectados por la actividad humana, con especial referencia a cambios en los procesos que determinan el funcionamiento del sistema Tierra" (Duarte, 2006: 23) 
[02] Por cambio climático se entiende la definición de la Convención Marco sobre Cambio Climático aprobada en la Cumbre de Río de 1992 y vigente en las negociaciones: "las alteraciones del clima que pueden ser atribuidas directa o indirectamente a las actividades humanas que modifiquen la composición de la atmósfera global y perturben así la variabilidad natural del clima"

[03] El término transición ha sido usado por numerosos autores, como Capra (1982), Meadows et al. (2006). Raskin et al (2006) o Hopkins (2008)

[04] Convención Marco de las Naciones Unidas sobre el Cambio Climático

[05] World Resources Institute, Estimates for Global Temperature Rise with INDCs above pre-industrial levels.

[06] http://www.un.org/sustainabledevelopment/es/objetivos-de-desarrollo-sostenible/, consultado el 30 de mayo de 2017

[07] Cabría preguntarse: ¿Desarrollo de qué? ¿Para quién? ¿Cuánto es demasiado? en un mundo con grandes desigualdades en un marco de convergencia donde sólo los países más pobres pueden tener justificado un incremento del impacto de la huella ecológica a la par que los países ricos reducen la suya

[08] Como afirman Meadows et al (2006: 397): "sería más seguro, y probablemente preferible, aprender a vivir una vida satisfactoria a cierta distancia seguir de los límites estimados del planeta, en vez de aspirar siempre a alcanzar el máximo físicamente posible".

[09] http://www.lavanguardia.com/natural/clima/20151212/30753432839/varapalo-de-los-cientificos-aldocumento-de-paris.html, consultado el 30 de mayo de 2017

[10] El observatorio de Observatorio Mauna Loa contabilizó el 21 de mayo de 2017 una concentración de 410,09 ppm de CO2, una cifra que se aleja de los 280 que existía antes de la época industrial

[11] En cuanto a la agenda temática de un periodismo en transición en torno al cambio climático es importante la disposición del periodista y de los medios. Normalmente los periodistas ambientales son los que introducen la sensibilidad por esta materia, a pesar de la reticencia de las estructuras comunicativas. En cierta manera son, muchas veces sin pretenderlo, pioneros culturales

[12] Es preciso "cambiar de gafas para mirar al mundo" y tratar desde otro prisma, el de la sostenibilidad y la equidad, temas como la energía, la economía, la tecnología, la propia información, la realidad virtual, la movilidad, el crecimiento, las necesidades humanas, el mundo laboral, la educación, el género, la publicidad, etc. (Herrero et al, 2011)

[13] Cambio Climático: Mitigación. Guía resumida del Quinto Informe de Evaluación del IPCC, Grupo de Trabajo III (2014). Ministerio de Agricultura Alimentación y Medio Ambiente, p. 7

[14] La búsqueda de un consenso por unanimidad ha rebajado el nivel de los borradores hasta puntos tan ilógicos como éste y supedita, en el presente y en el futuro, que cualquier disenso eche por tierra las tomas de medidas que se requieren para no traspasar los márgenes de seguridad señalados por la ciencia.

[15] http://www.efeverde.com/noticias/refugiados-climaticos-migrar/, consultado el 30 de mayo de 2017

[16] http://apps1.semarnat.gob.mx/dgeia/informe_12/05_atmosfera/cap5_2.html, consultado el 1 de junio de 2017

[17] Ségoléne Royal, en la COP 22, en http://www.un.org/sustainabledevelopment/blog/2016/11/the-eyes-of-theworld-are-upon-us-chair-of-un-conference-says-as-new-round-of-climate-talks-opens/, consultado el 30 de mayo de 2017 
[18] http://www.oei.es/divulgacioncientifica/?La-poblacion-mundial-superara-los, consultado el 30 de mayo de 2017

[19] Como expone el informe Cambio Global en España, "un poderoso mecanismo de conformación del comportamiento social a considerar es la publicidad que fomenta unas pautas consumistas directas, con sus derivaciones energéticas indirectas, muy lejos del comportamiento responsable. Por su presencia, persistencia e intensidad la influencia de la publicidad consumista tiene una enorme capacidad para contrarrestar los mensajes hacia el ahorro energético que eventualmente aparecen. Sería necesario un cambio muy drástico y profundo en este campo para neutralizar su influencia en el fomento del consumismo" (2011: 223)

[20] Tal como apunta el profesor Reig, "la Información en general y periodística en particular, es que, a diferencia de la información publicitaria, persuade y construye realidades en el receptor sin decirlo, sin reconocerlo" (1994: 16)

[21] Desde el IV Informe del IPCC (2007), "muchas tecnologías de energías renovables han demostrado mejoras sustanciales sobre su rendimiento y reducción de costos, y un mayor número de tecnologías de energías renovables han alcanzado un nivel de maduración para permitir un despliegue a escala significativa" (IPCC, 2014b: 69). Pero, ¿se puede mantener el actual nivel de consumo energético? Hay parámetros de otro paradigma que no son tenidos en cuenta por el IPCC. Creemos que no basta la eficiencia y la tecnología. Habría que sumarle una voluntad de decrecimiento material y energético, de una transformación cultural, de sobriedad compartida, de un cambio de paradigma con la sostenibilidad como eje

[22] Decadal changes calculated by CO 2 Earth with NOAA-ESRL annual mean CO2 concentrations at Mauna Loa, en https://www.co2.earth/, consultado el 30 de mayo de 2017

[23] "Es preciso conocer quién nos habla a través de los mensajes informativos y el poder que respalda a los medios de comunicación que nos acompañan a diario, y en los que depositamos en muchas ocasiones nuestra confianza, ya que esta estructura de poder influye decisivamente para que los mensajes tengan un contenido u otro, una intención u otra" (Reig, 1998: 11)

[24] Kumi Naidoo, Director de Greenpeace en El País, 16 de noviembre de 2009

[25] El Acuerdo invita al Grupo Intergubernamental de Expertos sobre el Cambio Climático a que presente, en 2018, un informe especial sobre los efectos que produciría un calentamiento global de $1,5^{\circ} \mathrm{C}$ con respecto a los niveles preindustriales y las trayectorias correspondientes que deberían seguir las emisiones mundiales de gases de efecto invernadero. Es una buena oportunidad de focalizar la atención en lo que supone este objetivo

[26] En los últimos meses estamos asistiendo cómo el presidente Donald Trump está generando una mayor cobertura mediática, apareciendo con connotaciones de "villano" climático por sus decisiones políticas

[27] En el ámbito de las políticas medioambientales y climáticas existen cuatro planteamientos consolidados y complementarios: mitigación, adaptación, evitación y restauración, en El Medio Ambiente en Europa. Estado y perspectiva 2015, p. 15

[28] El referente James Hansen considera como principal amenaza "el riesgo de que las placas de hielo puedan sufrir un forzamiento en este siglo que esté muy por encima de los rangos que consideramos naturales", en http://www.elespanol.com/ciencia/ecologia/20170613/223478220_0.html

[29] Herramienta que sirve para determinar el área de tierra y mar ecológicamente productiva que se requiere para proveer todos los recursos materiales y toda la energía consumidos, y también para poder absorber todos los residuos producidos por una población determinada y con el actual nivel tecnológico, sea donde sea que se encuentre esta área" (Rees y Wackernagel, 1996)

[30] Cantidad de gases de efecto invernadero emitidos a la atmósfera derivados de las actividades de producción o consumo de bienes y servicios de los seres humanos 
[31] Living Planet Report 2016, WWF, p.75

[32] http://www.footprintnetwork.org/, consultado el 30 de mayo de 2017

[33] https://www.co2.earth/co2-acceleration, consultado el 30 de mayo de 2017

- Recibido: 15 de junio de 2017

- Aceptado: 30 de junio de 2017

Ámbitos. Revista Internacional de Comunicación, n.37, edición de verano, 2017. 